\title{
Stratifin Indicates Worse Prognosis and Promotes Hepatocellular Carcinoma Proliferation, Migration, Invasion and EMT By Modulating Wnt/ $\beta$-Catenin Pathway
}

\section{Xiaoye Cheng}

The First Affiliated Hospital of Nanchang University

\section{Taiyuan Li}

The First Affiliated Hospital of Nanchang University

Jun Shi

The First Affiliated Hospital of Nanchang University

Shanping Ye ( $\square$ yeshanping123@sina.com )

The First Affiliated Hospital of Nanchang University

\section{Research Article}

Keywords: Stratifin, Hepatocellular carcinoma, Prognosis, EMT, Wnt/ $\beta$-catenin pathway

Posted Date: September 27th, 2021

DOl: https://doi.org/10.21203/rs.3.rs-842346/v1

License: (c) (1) This work is licensed under a Creative Commons Attribution 4.0 International License.

Read Full License 


\section{Abstract}

Background: Stratifin (SFN) is closely related to the tumor progression. However, the role of SFN in hepatocellular carcinoma (HCC) is still unknown. The purpose of this study is to investigate the clinical value, biological role and regulatory mechanisms of SFN in HCC.

Methods: Immunohistochemistry and RT-qPCR was used to explore SFN expression in HCC tissues and corresponding adjacent non-tumor tissues. The SFN expression profile data and clinical data of HCC patients were extracted from GEO, TCGA and Oncomine database. The univariate and multivariate analysis were used to investigate the prognosis value of the SFN gene in patients with HCC based on online database. The effects of SFN on HCC cell proliferation, migration, invasion was investigated by preforming CCK-8, colony formation, wound healing and Transwell assays. Xenograft nude mouse were used to observe the role of SFN on tumor growth. Western blotting was used to explore the genes associated with Epithelial mesenchymal transformation (EMT) and Wnt/ $\beta$-catenin signaling. The luciferase reported assay was used to validate the activity of Wnt signal pathway.

Results: In this study, we found SFN was upregulated in HCC cell lines and tissues. Clinically, SFN was positively associated with tumor size, degree of differentiation, TNM stage and vascular invasion. Survival analysis indicated that patients with high SFN levels had worse OS and DFS. SFN was an independent prognostic factor for HCC. Biologically, knockdown of SFN repressed tumor cell proliferation, migration, invasion, epithelial-to-mesenchymal transition (EMT) in vitro and tumor growth in vivo. Conversely, overexpression of SFN promoted these effects. Moreover, SFN promoted matrix metalloproteinases-2 (MMP-2) and MMP-9 expression. Mechanistically, SFN activated Wnt/ $\beta$-catenin pathway by promoting GSK-3 $\beta$ phosphorylation, decreasing $\beta$-catenin phosphorylation, promoting $\beta$ catenin nuclear translocation, increasing c-Myc expression and inhibiting Axin2 expression. Furthermore, the TOP/FOP-Flash reporter assays indicated that SFN overexpression or SFN knockdown obviously upregulated or down-regulated Wnt signaling activity.

Conclusions: SFN indicates worse survival in $\mathrm{HCC}$ and promotes $\mathrm{HCC}$ growth, migration, invasion and EMT by activating Wnt/ $\beta$-catenin pathway. Our results suggest SFN may become a prognostic factor and therapeutic target for patients with HCC in future.

\section{Background}

Liver cancer ranks sixth in cancer morbidity and fourth in cancer mortality worldwide in 2018[1-3]. Hepatocellular carcinoma (HCC) accounts for $75 \%$ - $85 \%$ of primary liver cancer, leading to nearly 745,000 patients deaths in the world every year $[1,4]$, seriously threatening the public health. The incidence of HCC is regionally heterogeneous, about $85 \%$ of HCC patients are estimated to happen in developing or less developed countries, among whom $72 \%$ of patients occur in Asia and more than $50 \%$ cases in China[1,5]. Unfortunately, most of HCC patients are at advanced stages when diagnosed and the incidence-tomortality ratio closes to $100 \%$ [6]. HCC surveillance (ultrasound and AFP) can help to detect tumor at an 
early stage when patients are potential to obtain the best curative outcomes [7]. For HCC patients, precise evaluation of prognosis is a key element for the treatment decision[8, 9]. Therefore, it is certainly needed to identify reliable biomarkers for evaluation of prognosis and targets for treatment in HCC patients[9]. Currently, more and more researches indicated that the abnormal expression of genes is closely correlative to the occurrence, development and prognosis of HCC, but the specific functions of most of them are still unclear[10, 11].

Stratifin (SFN) is a checkpoint protein of cell cycle and a regulatory factor of mitotic translation[12]. SFN is closely related to many cellular processes, especially for the regulation of cell cycle and signal transduction pathways[13]. Overexpression of SFN has been reported in many human tumors, such as ovarian cancer[14], breast cancer[15], lung cancer[12], HCC[16]. Conversely, downregulation of SFN has been observed in gliomas[13], nervous system[17], reproductive system[17]. Hu et al. have reported that upregulation of SFN was associated with worse prognosis in ovarian cancer[14]. Deng et al. indicated that SFN was an independent risk factor of prognosis for patients with gliomas[13]. But the role of SFN in HCC was rarely reported and its functions remained unclear.

In this study, we investigated the novel roles of SFN in HCC and found that SFN is up-regulated in HCC tissues and cell lines and is positively associated with tumor size, degree of differentiation, TNM stage, vascular invasion, and poor prognosis. SFN promotes HCC proliferation, migration, invasion, epithelial-tomesenchymal transition (EMT) by activating Wnt/ $\beta$-catenin pathway. Therefore, SFN is expected to be a therapeutic target and prognostic factor for HCC.

\section{Methods}

\section{Patients, clinical samples and follow-up}

A total of 34 pairs of HCC tissues and adjacent non-tumor tissues were obtained from HCC patients aged from 23 to 76 years, all of whom underwent radical resection between October 2017 and January 2018. The fresh specimens were stored in liquid nitrogen and embedded in paraffin respectively. The clinical data was collected from electronic medical record and the survival information was obtained by followup. The methods of follow-up were similar to the previous research [18]. All of the patients did not receive any adjuvant before operation and signed the written informed consent. Our study was compliance with the Helsinki Declaration and was approved by the institutional review of The First Affiliated Hospital of Nanchang University.

\section{Cell lines and cultures}

The HCC cell lines MHCC-97H, MHCC-97L were obtained from the Liver Cancer Institute of Fudan University (Shanghai, China). The HCC cell lines Huh7, SMMC-7721, HepG2 and the normal liver cell line L02 were obtained from the Chinese Academy of Sciences (Shanghai, China). All of them were cultured in Dulbecco's modified Eagle's medium (DMEM, Gibco, USA) containing 10\% fetal bovine serum (FBS, Gibco, 
USA), $100 \mathrm{U} / \mathrm{ml}$ penicillin and $100 \mathrm{ug} / \mathrm{ml}$ streptomycin (Gibco, USA). All cells were placed in an incubator with $5 \% \mathrm{CO}_{2}$ and humidified at $37^{\circ} \mathrm{C}$.

\section{RNA extraction and quantitative real-time PCR (RT-qPCR)}

Total RNA was obtained from the cultured cells or fresh-frozen tissues by using TRIzol Reagent (Invitrogen, USA) and reverse transcribed into cDNA by using PrimeScript RT reagent Kit with gDNA Eraser (Takara, Japan) in accordance with the manufacturer's instructions. RT-qPCR was performed in a StepOnePlus ${ }^{\mathrm{TM}}$ Real-Time PCR System (Applied Biosystems, USA) by using TB Green ${ }^{\mathrm{R}}$ Premix Ex Taq ${ }^{\mathrm{TM}}$ II (Tli RnaseH Plus) Kit (Takara, Japan). The $\beta$-actin was set as an internal control. All data were analyzed following the method of $2^{-} \triangle \triangle \mathrm{CT}$. The primers used in the current study were as follows: SFN: forward primer: 5'-TGACGACAAGAAGCGCATCAT-3', reverse primer: 5'- GTAGTGGAAGACGGAAAAGTTCA -3', $\beta$-actin was used as an internal control.

\section{Immunohistochemistry (IHC)}

The IHC was performed as described previously [19]. The sections of HCC tissues and adjacent nontumor tissues were incubated with primary antibody anti-SFN (ab193667, 1:600, Abcam) in $4{ }^{\circ} \mathrm{C}$ overnight. The IHC score was based on the staining intensity and the positive percentage of cells. The rate of positive cells was graded as follows: $0-5 \%$ was defined as 0 score, $6-25 \%$ as $1,26-50 \%$ as 2 , $51-75 \%$ as 3 , more than $75 \%$ as 4 . Moreover, the intensity of staining was scored as described next: 3 for strongly positive, 2 for moderately positive, 1 for weak and 0 for negative. The final score of IHC was equal to the score of staining intensity timing the score of positive percentage of cells. The final score $\geq$ 6 was defined as high SFN expression, otherwise it is regarded as low SFN expression. The IHC images were got with a microscope (Olympus, Japan).

\section{Establishment of overexpressing and knockdown HCC cells}

The knockdown and ectopic expression lentiviruses for SFN, and the corresponding control lentivirus were synthesized by HANBIO (Shanghai, China). The two shRNA sequences (names as: shSFN\#1 and shSFN\#2) and cDNA clone (names as: SFN) are presented in the Supplementary Table 1. The transfection was performed in cell lines according to the manufacturer's protocol. In brief, $1 \times 10^{5} \mathrm{MHCC}$ $97 \mathrm{H}$ or SMMC-7721 cells were seeded in 6-well plates in DMEM with $10 \% \mathrm{FBS}$. The cells were transfected when the cell confluence was about $60 \% .1 \mathrm{ml}$ fresh medium with $20 \mu$ lentivirus solution replaced the old medium. After $4 \mathrm{~h}$, another $1 \mathrm{ml}$ fresh medium added into each well. The medium was replaced after $24 \mathrm{~h}$. The stable transfected cell lines were selected by puromycin with final concentration of 2 $\mathrm{ug} / \mathrm{ml}$. The efficiency of transfection was verified by RT-qPCR and WB.

\section{Cell proliferation assay}

The cell proliferation was assayed using Cell Counting Kit-8 (CCK-8; Dojindo, Japan) according to the protocol of manufacturer. Briefly, $5 \times 10^{3} \mathrm{MHCC}-97 \mathrm{H}$ or SMMC-7721 cells were seeded into 96-well plates 
when it stable transfected lentivirus past $48 \mathrm{~h}, 5$ wells for per group. Then, at the time of $0,24,48,72,96$ $\mathrm{h}$, each well was added $10 \mu \mathrm{L}$ CCK-8 reagent, and the cells was incubated in incubator for $2 \mathrm{~h}$. At last, the multimode reader (TECAN SPARK 10M, Switzerland) was used to measure the absorbance at $450 \mathrm{~nm}$.

\section{Colony formation assays}

Infected cells were plated in 6-well plates, and the medium was replaced every five days. After two weeks, the cells were fixed by $4 \%$ paraformaldehyde and stained by $1 \%$ crystal violet. Then, the 6 -plates were washed with running water. The number of cell colony was counted on the pictures.

\section{Wound healing assay}

After transfected $48 \mathrm{~h}, \mathrm{MHCC}-97 \mathrm{H}$ or SMMC-7721 cells were seeded into 6-well plates. When the cells reached to $95 \%$ confluency, the scratch wound was got by a $200 \mu \mathrm{L}$ pipette tip through drawing lines on the surface cells in 6 -well plates. The images of wound healing at 0 and $24 \mathrm{~h}$ were photographed using microscope (10x) from each well.

\section{Migration and invasion assays (Transwell)}

The transwell chambers (Corning, USA) with the pore size at $8 \mu \mathrm{m}$ and 24-well plates were used to assay the cell migration and invasion assays. For the migration experiments, $5 \times 10^{4}$ cells were seeded into the upper chambers in $200 \mu \mathrm{L}$ DMED medium without FBS, while $700 \mu \mathrm{L}$ DMEM containing $20 \%$ FBS was added in the bottom plates. For the invasion experiments, $50 \mu \mathrm{L}$ Matrigel/DMEM (1:8, BD Biosciences, USA) was added into the upper transwell chambers. The other procedures were similar to the migration except for the number of cells $\left(1 \times 10^{5}\right.$ cells). After incubation for $24 \mathrm{~h}$, the chambers were fixed in the $4 \%$ paraformaldehyde and stained with $0.1 \%$ crystal violet. Then, the cells or Matrigel on the upper chambers were removed by cotton swab. HCC cells were counted from five random fields by using microscope (10 $\times)$.

\section{The xenograft mice models}

The 6-week-old male BALB/c nude mice purchased from Hunan SJA Laboratory Animal CO. LTD were used to explore the role of SFN on the tumor growth (six mice for each group). A total of $5 \times 10^{6}$ of HCC cells transfected with lentivirus in 100 ul DMEM medium containing $50 \%$ Matrigel were subcutaneously injected into the right upper flank regions of mice. The tumor length $(L)$ and width $(W)$ were measured every three days. The equation $1 / 2 \times L \times W^{2}$ was used to calculate the tumor volume. After 34 days, the mice were put into the euthanasia box and carbon dioxide was injected into the box at the rate of $30 \%$ of the volume of the euthanasia box per minute. After the animals stopped breathing and its pupils dilated, the next experiments could be carried out. The tumors were excised for further study. All animal researches were approved by the Medical Experimental Animal Care Commission of The First Affiliated Hospital of Nanchang University. 
The reporter plasmids encoding TOP-flash or FOP-flash with TCF/LEF DNA binding sites and control plasmids pTK-RL were purchased from Beyotime Biotechnology (China). Infected cells were plated in 24well plates, the pTK-RL and the flash plasmids were co-transfected into the cells. After $48 \mathrm{~h}$, the luciferase activity was analyzed as normalized to Renilla luciferase activity.

\section{Western blotting}

The total proteins from cells and tissues were isolated with RIPA buffer containing protease inhibitors. After centrifugation, the supernatants were collected for subsequently experiments. The NE-PER ${ }^{\mathrm{TM}}$ Nuclear and Cytoplasmic Extraction Reagents (Thermo Scientific, USA) were used to extract nuclear proteins. The BCA Protein Quantitation kit (Pierce, USA) was used to measure the protein concentration. A total of 30 $\mu \mathrm{g}$ protein from cells or tissues were separated by $8 \%-12 \%$ SDS-PAGE and transferred into the polyvinylidene fluoride (PVDF, Millipore, USA) membranes. The PVDF membranes were blocked with $5 \%$ skimmed milk for $1 \mathrm{~h}$ at $37^{\circ} \mathrm{C}$ and incubated with primary antibodies against SFN (ab193667, 1:1000, Abcam), $\beta$-actin (ab8226, 1:1000, Abcam), E-cadherin (\#3195, 1:1000, Cell Signaling Technology), Ncadherin(\#13116, 1:1000, Cell Signaling Technology), Vimentin(\#5741, 1:1000, Cell Signaling Technology), MMP-2 (ab92536, 1:1000, Abcam), MMP-9 (ab76003, 1:1000, Abcam), $\beta$-Catenin(\#8480, 1:1000, Cell Signaling Technology), Non-phospho (Active) $\beta$-Catenin(\#19807, 1:1000, Cell Signaling Technology), GSK-3ß(\#12456, 1:1000, Cell Signaling Technology), Phospho-GSK-3ß (Ser9) (\#5558, 1:1000, Cell Signaling Technology), Axin2(ab109307, 1:1000, Abcam), and c-Myc(ab32072, 1:1000, Abcam) overnight at $4{ }^{\circ} \mathrm{C}$. After washed with Tris-buffered saline Tween buffer, the membranes were incubated with HPR-rabbit (SA00001-2, 1:5000, proteintech) or HPR-mouse (SA00001-1, 1:5000, proteintech) at $37{ }^{\circ} \mathrm{C}$ for $1 \mathrm{~h}$. The intensity of band was quantified by ImageJ (National Institutes of Health, USA) and the specific details were described as previously [20].

\section{SFN expression in TCGA, GEO and Oncomine databases}

The gene expression profiles of liver hepatocellular carcinoma (LIHC) obtained from RNA-Seq HTSeqFPKM platform were downloaded from GDC Data Portal of The Cancer Genome Atlas (TCGA) (https://portal.gdc.cancer.gov/repository) consisted of $374 \mathrm{HCC}$ tissues and 50 adjacent non-tumor tissues. Limma package (R) was used to identify SFN between HCC and non-tumor tissues[21].

For Gene Expression Omnibus (GEO) datasets (https://www.ncbi.nlm.nih.gov/geo/), we downloaded ten expression microarray profiles (GSE: 14520, 25097, 45114, 45436, 55092, 57555, 60502, 76427, 77314, 101728) including HCC tissues and non-tumor tissues. Except for the SFN expression of GSE77314 was directly calculated by the XLSX data downloaded from GEO datasets, difference of SFN expression between tumor and non-tumor tissues of others GEO series were performed by Limma package (R)[21].

For Oncomine database (https://www.oncomine.org/), there were four studies compared SFN expression between hepatocellular carcinoma and normal samples. We comprehensively analyzed four studies with following threshold: $p$-Value $\leq 1 \mathrm{E}-4$, fold change $\geq 2$, gene ranks in the top $10 \%$. 


\section{Prognosis Analysis based on online database}

To investigate the prognosis value of the SFN gene in patients with $\mathrm{HCC}$, we conducted survival analysis and univariate and multivariate analysis. The univariate and multivariate Cox regression analysis of OS were performed by "survival" and "survminer" packages (https://cran.r-project.org/web/packages/) based on $\mathrm{R}$ language. The data downloaded from Kaplan-Meier plotter database (http://kmplot.com/analysis/) was used for survival analysis[22]. The cut-off value was the median expression of SFN in HCC patients. Graphpad prism software (Version 7) was used to plot all survival curves.

\section{Statistical analysis}

The data statistical analyses in this paper were performed using SPSS 22.0 software and GraphPad Prism 7. Mann-Whitney U-test, student $t$ test or Chi-square test, and Spearman's rank analysis was performed according to the type of variables. A two-tailed $P \otimes 0.05$ were considered as statistically significant.

\section{Results}

\section{SFN is overexpressed in human HCC cell lines and tissues}

Firstly, we explored the expression levels of SFN in the HCC cell lines and normal cell L02. Both SFN mRNA and protein was up-regulated in all HCC cell lines compared to L02 cell (Fig. 1A and 1C). Then, we examined the SFN mRNA and protein levels in clinical fresh HCC tissues. The HCC tissues had higher mRNA and protein expression levels of SFN than that in adjacent nontumor liver tissues (Fig. 1B and 1D). Moreover, the expression of SFN protein was also higher in HCC tissues than that in the related adjacent non-tumor liver tissues as detected by IHC (Fig. 1E and 1F). To further confirm the level of SFN expression, we also analyzed data derived from the TCGA, GEO, Oncomine database. As shown in Fig. 1G, the SFN mRNA based on TCGA was significantly up-regulated in HCC tissues $(P \otimes 0.05)$. In Oncomine database, the SFN gene was also obviously overexpressed in HCC tissues in Wurmbach Liver dataset, Mas Liver dataset, Roessler Liver 2 dataset, Roessler Liver dataset (all $P \otimes 0.05$, Fig. 1H, $1 \mathrm{l}, 1 \mathrm{~J}$ and $1 \mathrm{~K}$ ). As shown in Supplement Fig. 1A, the meta-analysis of four Oncomine analyses was also validated the high SFN expression in tumors $(P<0.05)$. Furthermore, we analyzed the expression of SFN in GEO databases. Consistent with the results of TCGA and Oncomine database, the SFN gene was also up-regulated in ten GEO series (GSE: 14520, 25097, 45114, 45436, 55092, 57555, 60502, 76427, 77314, 101728, shown in Supplement Fig. $1 \mathrm{~B}$ to $1 \mathrm{~K}$, all $P \otimes 0.05)$. In conclusion, all the above results indicated that $S F N$ is significantly up-regulated in human HCC cell lines and tissues.

SFN was closely associated with aggressive clinicopathological features and poor prognosis in HCC patients 
To further study the clinicopathological significance of SFN, we analyzed the correlation between SFN expression and clinicopathological characteristics in $34 \mathrm{HCC}$ patients. Patients were divided into high and low SFN expression groups based on the median level of SFN expression by RT-qPCR or IHC. In the RTqPCR cohort, the SFN expression is bound up with the tumor size, degree of differentiation, TNM stage, and vascular invasion ( $P \otimes 0.05$, Table 1$)$. In the IHC cohort, SFN is also closely related with the degree of differentiation $(P=0.039)$, TNM stage $(P=0.001)$ and vascular invasion $(P=0.005)$. However, the tumor size showed no difference between the two groups in accordance with the score of $\operatorname{IHC}(P=0.080)$.

To evaluate the prognostic value of SFN in HCC patients, we compared the difference of overall survival (OS) and disease-free survival (DFS) between high and low SFN group in HCC patients. As we expected, SFN high group was associated with worse 3 years OS and DFS as compared with low SFN group ( $P$ $\nabla 0.05$, Fig. 2A and 2B, respectively). We further performed survival analysis using the Kaplan-Meier plotter online database. The results indicated that SFN over-expression in HCC tumor was significantly associated with worse OS, progression-free survival (PFS), relapse-free survival (RFS), and disease-free survival (DSS) in HCC patients ( all log rank $P \otimes 0.05$, Fig. 2C, 2D, 2E and 2F).

Furthermore, we evaluate the prognostic value of SFN in HCC cases by univariate and multivariate analysis. The clinicopathological data of 374 cases of HCC were obtained from TCGA data. Due to the incomplete clinicopathological data of many cases, we selected some common features (age, gender, tumor grade, TNM stage, T, N, M) for further analysis. Exclude unavailable data including unknown, missing, Tx, Nx and Mx data. Finally, 212 HCC patients were enrolled in this study. The univariate analysis indicated that TNM stage, T, M, SFN expression were significantly associated with OS $(P=6.71 \mathrm{e}-$ 07, 5.65e-07, 0.0227 and 3.02e-06, respectively, Table 2). As shown in Table 2, multivariable analysis indicated that SFN was an independent prognostic factor for OS $(P=0.0005)$. Therefore, we suggested that SFN could affect the progress of HCC and may be regarded as a novel prognostic marker for HCC.

\section{SFN promotes HCC cells proliferation, migration, invasion, growth}

To explore the biological function of SFN in the progress of HCC, we up regulated SFN expression by ectopic expression lentiviruses in the SMMC-7721 (named: SMMC-7721-Lv-SFN) cell with the lowest SFN expression, and we down-regulated SFN expression by knockdown expression lentiviruses in the MHCC97H (named: MHCC-97H-Lv-shSFN) cell with the highest SFN expression. The RT-qPCR and Western blotting were used to estimate the knockdown efficiency (Fig. 3A, 3B, 3D and 3E). CCK-8 and colony formation assays were used to explore the proliferative capacity of two cell lines. We found that the overexpression of SFN promoted colony formation ability and proliferation (all $P \otimes 0.05$, Fig. 3C, 3F and $3 \mathrm{~K}$ ). Conversely, the knockdown of SFN inhibited colony formation ability and proliferation (all $P \otimes 0.05$, Fig. 3C, 3F and 3K). Furthermore, we observed that migration ability of SMMC-7721-Lv-SFN cells was significantly enhanced while the migration ability of MHCC-97H-Lv-shSFN cells was obviously inhibited (Fig. 4G and 4I) in wound healing assay. Similarly, transwell assays displayed the same results with wound healing assay (Fig. $4 \mathrm{H}$ and $4 \mathrm{~J}$ ). Importantly, we evaluated the effect of SFN on tumor growth in vivo. As we expected, we found that the SMMC-7721-Lv-SFN cells obviously enhanced the abilities of 
tumor growth compared with SMMC-7721-control cells. The MHCC-97H-Lv-shSFN cells significantly inhibited the abilities of tumor growth (Fig. 4L). The tumor weight of the SMMC-7721-Lv-SFN cells were markedly higher than that of SMMC-7721-control cells while the tumor weight of MHCC-97H-Lv-shSFN cells were significantly lower than that of MHCC-97H- control cells (Fig. 4M to 4P). Taken together, these data demonstrated that SFN promoted HCC cells proliferation, migration, invasion in vitro and tumor growth in vivo.

\section{SFN induces EMT and activates Wnt/ $\beta$-catenin signaling}

EMT is an important process for tumor progression. To further explore the mechanism of SFN in HCC progression, we examined the markers of EMT. We found that the ectopic expression of SFN in SMMC7721 resulted in increased expression of $\mathrm{N}$-cadherin and Vimentin (mesenchymal markers) and decreased expression of E-cadherin (epithelial marker), while SFN knockdown in MHCC-97H cells led to the adverse results as shown by western blot (Fig. 4A and 4B). As we known, Wnt/ $\beta$-catenin signaling is a crucial pathway for HCC progression, and is closely related with the process of EMT[23]. Therefore, we speculated that SFN may also activate Wnt/ $\beta$-catenin signaling to promote HCC progression. To verify the hypothesis, we explored the role of SFN on the expression of key markers of the Wnt/ $\beta$-catenin signaling pathway $[24,25]$. In SFN-overexpressing cells, we observed that $\beta$-Catenin, non-phospho (active) $\beta$-Catenin, phospho-GSK-3 $\beta$ (Ser9) were up-regulated, and GSK-3 $\beta$ was down-regulated (Fig. 4C). Whereas, opposing results showed from the SFN knockdown cells (Fig. 4D). As we known, the translocation into nuclear of $\beta$-Catenin is the biomarker for Wnt/ $\beta$-catenin signaling activation[26]. We also detected the protein expression levels of $\beta$-Catenin in nuclear, and found that SFN overexpression promoted $\beta$-Catenin accumulation in nuclear and SFN knockdown exhibited contrary trend (Fig. 4E and 4F). Furthermore, we analyzed several genes which are associated with cell invasion and EMT. The results showed that MMP2, MMP-9 were upregulated in SFN-overexpressing cells and downregulated in SFN knockdown cells (Fig. $4 C$ and $4 D$ ). Taken together, the results indicate that SFN induces EMT and activates Wnt/ $\beta$-catenin signaling.

\section{Discussion}

HCC is a common cancer type and cause of cancer-related death around the world, particularly in the Asia-Pacific region [27]. As we know, it is also a cancer with heterogeneous feature and the clinical outcome is variable after treatment. Over the past few years, considerable researches have been performed to investigate prognostic markers for $\mathrm{HCC}[28]$. It has been reported that the high C-reactive protein levels at the time of diagnosis was associated with worse survival of HCC patients[29]. In the current study, we find that SFN is overexpression in HCC, and is significantly related to aggressive clinicopathological features and worse survival of patients with HCC. Moreover, our results indicate that SFN plays important roles in HCC proliferation, migration, invasion, growth and induces EMT by activating Wnt/ $\beta$-catenin signaling. Therefore, SFN may be a novel oncogene in HCC and is expected to be a therapeutic target and prognostic factor for patients with HCC. 
SFN is a member from conserved protein family of 14-3-3 and plays important functions in various biological processes[30]. Over-expression of SFN has been reported in human malignant tumors. SFN is also a prognostic factor for many malignant tumors, such as ovarian cancer[14], gliomas[13] and gallbladder cancer[31]. As expected, SFN was up-regulated in HCC patients and associated with aggressive clinicopathological features. Moreover, we observed that patients with high SFN expression were related with worse survival than that with low SFN expression. We further validated the result in public databases. The TCGA, GEO, and Oncomine database contains gene expression and clinical data and are the best cancer genomics programs, which has greatly promoted cancer research [32-34]. As shown in the Fig. 2A to 2J, the gene expression data in GEO database showed the higher expression of SFN in HCC than that in non-tumor tissues. Our results were consistent with previous studies[35, 36]. What's more, we investigated the prognosis value by using the Kaplan-Meier plotter online database and found that SFN over-expression in HCC tumor was significantly associated with worse OS, PFS, RFS, and DSS in HCC patients. Our data suggested that SFN may promote HCC prognosis. Previous researches have referred to the potential role of SFN in tumors $[37,38]$.

As evidenced by the above analysis, we speculated that SFN could affect the progress of HCC. But to date, the functions of SFN in HCC has not been verified. Then, we performed several assays to explore the roles of SFN in HCC. Previous studies indicated that SFN was closely correlated with cell cycle, apoptosis, signal transduction and protein trafficking[39]. The results of functional experiments demonstrated that SFN overexpression could obviously promotes HCC cells proliferation, migration, invasion, while SFN knockdown in HCC cells displayed opposing trend. Our study also revealed that SFN could enhance the growth capacities of HCC in vivo. EMT is vital in cancer development and progression[40]. EMT promotes tumor cells to acquire mesenchymal phenotype and ability of migration and invasion[40]. Our results indicated that silencing SFN suppressed the process of EMT and upregulating SFN promoted it. Our data further enriched the understanding of the biological role of SFN in HCC progression.

In the current study, we further illustrated that SFN facilitated HCC cells proliferation, migration, invasion, growth, and EMT by Wnt/ $\beta$-catenin signaling activation. Many researches have indicated that Wnt/ $\beta$ catenin signaling plays an important function in HCC progression[41]. $\beta$-catenin and Gsk-3 $\beta$ are key components of $W n t / \beta$-catenin signaling pathway[25, 41]. As we known, the levels of stable $\beta$-catenin play a key function in the output $W n t / \beta$-catenin signal[24]. Our results showed that the protein expression levels of $\beta$-Catenin, Non-phospho (Active) $\beta$-Catenin, and Phospho-GSK-3 $\beta$ (Ser9) in SFN-overexpressing cells were up-regulated. Whereas, opposing results showed from the SFN knockdown cells. To further confirmed the function of SFN on Wnt/ $\beta$-catenin pathway, we also examined the protein levels of $\beta$ Catenin in nuclear, and found that SFN overexpression promoted $\beta$-Catenin accumulation in nuclear and SFN knockdown exhibited contrary trend. These results may help us understand the relationship of SFN and $\mathrm{Wnt} / \beta$-catenin signaling. However, this is just a preliminary exploration and deeper mechanism need to be further investigated.

\section{Conclusions}


In conclusion, SFN is overexpressed in HCC tissues and cell lines, and is correlated with tumor size, differentiation, vascular invasion and TNM stage. The high SFN expression was obviously associated with worse survival. SFN is an oncogene in HCC, affecting the ability of cell proliferation, migration, invasion and growth. Moreover, SFN promoted the process of EMT and activated the Wnt/ $\beta$-catenin signaling pathway. The results of current analysis may provide new evidence for the prognosis and target therapy value of SFN. However, the underlying mechanism of SFN in HCC progression needs further study.

\section{Abbreviations}

AFP: Alpha-fetoprotein; CCK-8: Cell Counting Kit-8; DEGs: Differentially expressed genes; DFS: Diseasefree survival; DMEM: Dulbecco's modified Eagle's medium; DSS: Disease-specific survival; EMT: Epithelialmesenchymal transition; FBS: Fetal bovine serum; GEO: Gene Expression Omnibus; HCC: Hepatocellular carcinoma; IHC: Immunohistochemistry; LIHC: liver hepatocellular carcinoma; OS: overall survival; PFS: Progression free survival; RFS: Relapse-free survival; RT-qPCR: Quantitative real-time PCR; SFN: Stratifin; TCGA: The Cancer Genome Atlas.

\section{Declarations}

\section{Ethics approval and consent to participate}

The patients study protocol was approved by the institutional review of The First Affiliated Hospital of Nanchang University and the study compliance with the Helsinki Declaration. Human experiments were carried out under protocol as guidelines of the institutional review of The First Affiliated Hospital of Nanchang University. Written informed consents were obtained from all of the patients. All animal researches were approved by the Medical Experimental Animal Care Commission of The First Affiliated Hospital of Nanchang University. Animal experiments were carried out under protocol as guidelines of the Medical Experimental Animal Care Commission of The First Affiliated Hospital of Nanchang University.

\section{Consent for publication}

Not applicable

\section{Availability of data and materials}

The gene expression profiles of liver hepatocellular carcinoma (LIHC) obtained from RNA-Seq HTSeqFPKM platform were downloaded from GDC Data Portal of The Cancer Genome Atlas (TCGA) (https://portal.gdc.cancer.gov/repository) .The GSE datasets (GSE: 14520, 25097, 45114, 45436, 55092, $57555,60502,76427,77314,101728$ ) were downloaded from GEO databases (https://www.ncbi.nlm.nih.gov/geo/), 
The Oncomine database (https://www.oncomine.org/) was used for meta-analysis of SFN expression. Access to the database can be obtained from the corresponding author on reasonable request.

\section{Competing interests}

The authors declare that they have no competing interests.

\section{Funding}

Not applicable.

\section{Authors' Contributions}

SY and XC designed the study; XC contributed to downloaded the datas; XC and SY conducted the experiments and collected data; SY, XC, TL and JS analyzed the data; SY, XC, TL wrote the manuscript; SY and $\mathrm{XC}$ proofread and revised the manuscript. All authors read and approved the final manuscript.

\section{Acknowledgements}

Not applicable

\section{References}

1. Bray F, Ferlay J, Soerjomataram I, Siegel RL, Torre LA, Jemal A: Global cancer statistics 2018: GLOBOCAN estimates of incidence and mortality worldwide for 36 cancers in 185 countries. $C A$ Cancer J Clin 2018, 68(6):394-424.

2. Wang H, Naghavi M, Allen C, Barber RM, Bhutta ZA, Carter A, Casey DC, Charlson FJ, Chen AZ, Coates $\mathrm{MM}$ et al: Global, regional, and national life expectancy, all-cause mortality, and cause-specific mortality for 249 causes of death, 1980-2015: a systematic analysis for the Global Burden of Disease Study 2015. The Lancet 2016, 388(10053):1459-1544.

3. Villanueva A: Hepatocellular Carcinoma. N Engl J Med 2019, 380(15):1450-1462.

4. Yang JD, Roberts LR: Hepatocellular carcinoma: A global view. Nat Rev Gastroenterol Hepatol 2010, 7(8):448-458.

5. Tang A, Hallouch O, Chernyak V, Kamaya A, Sirlin CB: Epidemiology of hepatocellular carcinoma: target population for surveillance and diagnosis. Abdom Radiol (NY) 2018, 43(1):13-25.

6. Singal AG, Lampertico P, Nahon P: Epidemiology and surveillance for hepatocellular carcinoma: New trends. J Hepatol 2020, 72(2):250-261.

7. Singal AG, Pillai A, Tiro J: Early detection, curative treatment, and survival rates for hepatocellular carcinoma surveillance in patients with cirrhosis: a meta-analysis. PLoS Med 2014, 11(4):e1001624.

8. Zhang B, Finn RS: Personalized Clinical Trials in Hepatocellular Carcinoma Based on Biomarker Selection. Liver Cancer 2016, 5(3):221-232. 
9. Black AP, Mehta AS: The search for biomarkers of hepatocellular carcinoma and the impact on patient outcome. Curr Opin Pharmacol 2018, 41:74-78.

10. Ding ZY, Jin GN, Wang W, Chen WX, Wu YH, Ai X, Chen L, Zhang WG, Liang HF, Laurence A et al: Reduced expression of transcriptional intermediary factor 1 gamma promotes metastasis and indicates poor prognosis of hepatocellular carcinoma. Hepatology 2014, 60(5):1620-1636.

11. Xia L, Huang W, Tian D, Zhu H, Zhang Y, Hu H, Fan D, Nie Y, Wu K: Upregulated FoxM1 expression induced by hepatitis $B$ virus $X$ protein promotes tumor metastasis and indicates poor prognosis in hepatitis B virus-related hepatocellular carcinoma. J Hepatol 2012, 57(3):600-612.

12. Kim Y, Shiba-Ishii A, Nakagawa T, lemura SI, Natsume T, Nakano N, Matsuoka R, Sakashita S, Lee S, Kawaguchi A et al: Stratifin regulates stabilization of receptor tyrosine kinases via interaction with ubiquitin-specific protease 8 in lung adenocarcinoma. Oncogene 2018, 37(40):5387-5402.

13. Deng J, Gao G, Wang $L$, Wang T, Yu J, Zhao Z: Stratifin expression is a novel prognostic factor in human gliomas. Pathol Res Pract 2011, 207(11):674-679.

14. Hu Y, Zeng Q, Li C, Xie Y: Expression profile and prognostic value of SFN in human ovarian cancer. Biosci Rep 2019, 39(5).

15. Ko S, Kim JY, Jeong J, Lee JE, Yang WI, Jung WH: The role and regulatory mechanism of 14-3-3 sigma in human breast cancer. J Breast Cancer 2014, 17(3):207-218.

16. Zhang Y, Li Y, Lin C, Ding J, Liao G, Tang B: Aberrant upregulation of 14-3-3sigma and EZH2 expression serves as an inferior prognostic biomarker for hepatocellular carcinoma. PLoS One 2014, 9(9):e107251.

17. Winter $M$, Lodygin D, Verdoodt B, Hermeking H: Deletion of 14-3-3sigma sensitizes mice to DMBA/TPA-induced papillomatosis. Oncotarget 2016, 7(30):46862-46870.

18. Zhong X, Kan A, Zhang W, Zhou J, Zhang H, Chen J, Tang S: CBX3/HP1gamma promotes tumor proliferation and predicts poor survival in hepatocellular carcinoma. Aging (Albany NY) 2019, 11(15):5483-5497.

19. Wang R, Yu Z, Chen F, Xu H, Shen S, Chen W, Chen L, Su Q, Zhang L, Bi J et al: miR-300 regulates the epithelial-mesenchymal transition and invasion of hepatocellular carcinoma by targeting the FAK/PI3K/AKT signaling pathway. Biomed Pharmacother 2018, 103:1632-1642.

20. Qu W, Wen X, Su K, Gou W: MiR-552 promotes the proliferation, migration and EMT of hepatocellular carcinoma cells by inhibiting AJAP1 expression. J Cell Mol Med 2019, 23(2):1541-1552.

21. Ritchie ME, Phipson B, Wu D, Hu Y, Law CW, Shi W, Smyth GK: limma powers differential expression analyses for RNA-sequencing and microarray studies. Nucleic Acids Res 2015, 43(7):e47.

22. Menyhart O, Nagy A, Gyorffy B: Determining consistent prognostic biomarkers of overall survival and vascular invasion in hepatocellular carcinoma. $R$ Soc Open Sci 2018, 5(12):181006.

23. Teeuwssen M, Fodde R: Wnt Signaling in Ovarian Cancer Stemness, EMT, and Therapy Resistance. $J$ Clin Med 2019, 8(10).

24. Clevers H, Nusse R: Wnt/beta-catenin signaling and disease. Cell 2012, 149(6):1192-1205. 
25. Russell JO, Monga SP: Wnt/beta-Catenin Signaling in Liver Development, Homeostasis, and Pathobiology. Annu Rev Pathol 2018, 13:351-378.

26. Zhang T, Ma Z, Liu L, Sun J, Tang H, Zhang B, Zou Y, Li H: DDX39 promotes hepatocellular carcinoma growth and metastasis through activating Wnt/beta-catenin pathway. Cell Death Dis 2018, 9(6):675.

27. Zhu RX, Seto WK, Lai CL, Yuen MF: Epidemiology of Hepatocellular Carcinoma in the Asia-Pacific Region. Gut Liver 2016, 10(3):332-339.

28. Burkhart RA, Ronnekleiv-Kelly SM, Pawlik TM: Personalized therapy in hepatocellular carcinoma: Molecular markers of prognosis and therapeutic response. Surg Oncol 2017, 26(2):138-145.

29. Sieghart W, Pinter M, Hucke F, Graziadei I, Schöniger-Hekele M, Müller C, Vogel W, Trauner M, PeckRadosavljevic M: Single determination of C-reactive protein at the time of diagnosis predicts longterm outcome of patients with hepatocellular carcinoma. Hepatology 2013, 57(6):2224-2234.

30. Chan TA, Hermeking H, Lengauer C, Kinzler KW, Vogelstein B: 14-3-3Sigma is required to prevent mitotic catastrophe after DNA damage. Nature 1999, 401(6753):616-620.

31. Sirivatanauksorn V, Dumronggittigule W, Dulnee B, Srisawat C, Sirivatanauksorn Y, Pongpaibul A, Masaratana P, Somboonyosdech C, Sripinitchai S, Kositamongkol P et al: Role of stratifin (14-3-3 sigma) in adenocarcinoma of gallbladder: A novel prognostic biomarker. Surg Oncol 2020, 32:5762.

32. Hutter C, Zenklusen JC: The Cancer Genome Atlas: Creating Lasting Value beyond Its Data. Cell 2018, 173(2):283-285.

33. Chen $Y, L i Y$, Narayan R, Subramanian A, Xie X: Gene expression inference with deep learning. Bioinformatics 2016, 32(12):1832-1839.

34. Rhodes DR, Yu J, Shanker K, Deshpande N, Varambally R, Ghosh D, Barrette T, Pandey A, Chinnaiyan AM: ONCOMINE: a cancer microarray database and integrated data-mining platform. Neoplasia 2004, 6(1):1-6.

35. Reis H, Putter C, Megger DA, Bracht T, Weber F, Hoffmann AC, Bertram S, Wohlschlager J, Hagemann $S$, Eisenacher $M$ et al: A structured proteomic approach identifies 14-3-3Sigma as a novel and reliable protein biomarker in panel based differential diagnostics of liver tumors. Biochim Biophys Acta 2015, 1854(6):641-650.

36. Liu CC, Jan YJ, Ko BS, Wu YM, Liang SM, Chen SC, Lee YM, Liu TA, Chang TC, Wang J et al: 14-33sigma induces heat shock protein 70 expression in hepatocellular carcinoma. BMC Cancer 2014, 14:425.

37. Huang Y, Yang M, Huang W: 14-3-3 sigma: A potential biomolecule for cancer therapy. Clin Chim Acta 2020, 511:50-58.

38. Neal CL, Yu D: 14-3-3zeta as a prognostic marker and therapeutic target for cancer. Expert Opin Ther Targets 2010, 14(12):1343-1354.

39. Smith AJ, Daut J, Schwappach B: Membrane proteins as 14-3-3 clients in functional regulation and intracellular transport. Physiology (Bethesda) 2011, 26(3):181-191. 
40. Dongre A, Weinberg RA: New insights into the mechanisms of epithelial-mesenchymal transition and implications for cancer. Nat Rev Mol Cell Biol 2019, 20(2):69-84.

41. Perugorria MJ, Olaizola P, Labiano I, Esparza-Baquer A, Marzioni M, Marin JJG, Bujanda L, Banales JM: Wnt-beta-catenin signalling in liver development, health and disease. Nat Rev Gastroenterol Hepatol 2019, 16(2):121-136.

\section{Tables}

Table 1 Clinicopathological features of HCC patients between SFN high and low expression cohorts 


\begin{tabular}{|c|c|c|c|c|c|c|}
\hline \multirow[t]{2}{*}{ Features } & \multicolumn{2}{|l|}{ qRT-PCR } & \multirow[t]{2}{*}{$P$ value } & \multicolumn{2}{|l|}{$\mathrm{IHC}$} & \multirow[t]{2}{*}{$P$ value } \\
\hline & $\begin{array}{l}\text { Low }(n= \\
17)\end{array}$ & $\begin{array}{l}\text { High }(n= \\
17)\end{array}$ & & $\begin{array}{l}\text { Low }(n= \\
17)\end{array}$ & $\begin{array}{l}\text { High }(n= \\
17)\end{array}$ & \\
\hline Gender, n & & & 0.656 & & & 0.175 \\
\hline Male & 15 & 13 & & 12 & 16 & \\
\hline Female & 2 & 4 & & 5 & 1 & \\
\hline Age, years & & & 0.732 & & & 0.084 \\
\hline$\leq 50$ & 7 & 9 & & 5 & 11 & \\
\hline$\otimes 50$ & 10 & 8 & & 12 & 6 & \\
\hline HBV infection, $n$ & & & 1.000 & & & 1.000 \\
\hline Positive & 16 & 15 & & 15 & 16 & \\
\hline Negtive & 1 & 2 & & 2 & 1 & \\
\hline AFP, ng/ml & & & 0.166 & & & 1.000 \\
\hline$\leq 400$ & 7 & 12 & & 9 & 10 & \\
\hline$\nabla 400$ & 10 & 5 & & 8 & 7 & \\
\hline Tumor number, $\mathrm{n}$ & & & 1.000 & & & 0.227 \\
\hline Solitary & 16 & 15 & & 17 & 14 & \\
\hline Multiple & 1 & 2 & & 0 & 3 & \\
\hline Tumor size, cm & & & $0.013 *$ & & & 0.080 \\
\hline$\leq 5$ & 14 & 6 & & 13 & 7 & \\
\hline$\otimes 5$ & 3 & 11 & & 4 & 10 & \\
\hline Vascular invasion, $\mathrm{n}$ & & & $0.037 *$ & & & $0.005^{*}$ \\
\hline Yes & 4 & 11 & & 3 & 12 & \\
\hline None & 13 & 6 & & 14 & 5 & \\
\hline $\begin{array}{l}\text { Tumor } \\
\text { differentiation, } n\end{array}$ & & & $0.003^{*}$ & & & $0.039 *$ \\
\hline Well/moderate & 17 & 9 & & 16 & 10 & \\
\hline Poor & 0 & 8 & & 1 & 7 & \\
\hline Liver cirrhosis, $\mathrm{n}$ & & & 0.708 & & & 0.259 \\
\hline Yes & 13 & 11 & & 10 & 14 & \\
\hline
\end{tabular}




\begin{tabular}{|lccccc|} 
None & 4 & 6 & 7 & 3 & 0.001* \\
\hline AJCC TNM stage, $n$ & & & $\mathbf{0 . 0 1 3 *}^{*}$ & & \\
\hline I/ II & 14 & 6 & 15 & 5 & \\
\hline III/ IV & 3 & 11 & 2 & 12 \\
\hline
\end{tabular}

AFP: Alpha-fetoprotein; AJCC: American Joint Committee on Cancer; HBV: Hepatitis B virus; HCC. Hepatocellular Carcinoma; SFN: stratifin. ${ }^{*} \otimes \square 0.05$. Fisher exact test was used in all analysis.

Table 2 Univariate and multivariate survival analysis of OS in HCC patients

\begin{tabular}{|c|c|c|c|c|c|c|}
\hline \multirow[t]{2}{*}{ Variable } & \multicolumn{2}{|c|}{ Univariate analysis } & \multirow[t]{2}{*}{$P$ value } & \multicolumn{2}{|c|}{ Multivariate analysis } & \multirow[t]{2}{*}{$P$ value } \\
\hline & $\mathrm{HR}$ & $95 \% \mathrm{Cl}$ & & $\mathrm{HR}$ & $95 \% \mathrm{Cl}$ & \\
\hline Age & 1.006 & $0.987-1.025$ & 0.538 & 1.012 & $0.991-1.033$ & 0.275 \\
\hline Gender & 0.845 & $0.516-1.385$ & 0.505 & 1.022 & $0.585-1.787$ & 0.938 \\
\hline Grade & 0.996 & $0.726-1.366$ & 0.979 & 1.159 & $0.821-1.636$ & 0.403 \\
\hline AJCC TNM & 1.921 & $1.485-2.485$ & 0.000 & 2.041 & $0.642-6.488$ & 0.227 \\
\hline $\mathrm{T}$ & 1.844 & $1.451-2.344$ & 0.000 & 0.937 & $0.320-2.745$ & 0.906 \\
\hline N & 2.018 & $0.492-8.279$ & 0.330 & 1.080 & $0.125-9.310$ & 0.944 \\
\hline$M$ & 3.867 & $1.208-12.374$ & 0.023 & 0.755 & $0.191-2.987$ & 0.689 \\
\hline SFN expression & 1.009 & $1.005-1.013$ & 0.000 & 1.008 & $1.004-1.013$ & 0.000 \\
\hline
\end{tabular}

Cl: Confidence interval; HCC. Hepatocellular carcinoma; HR: Hazard ratio; OS. Overall survival; SFN: Stratifin.

\section{Figures}



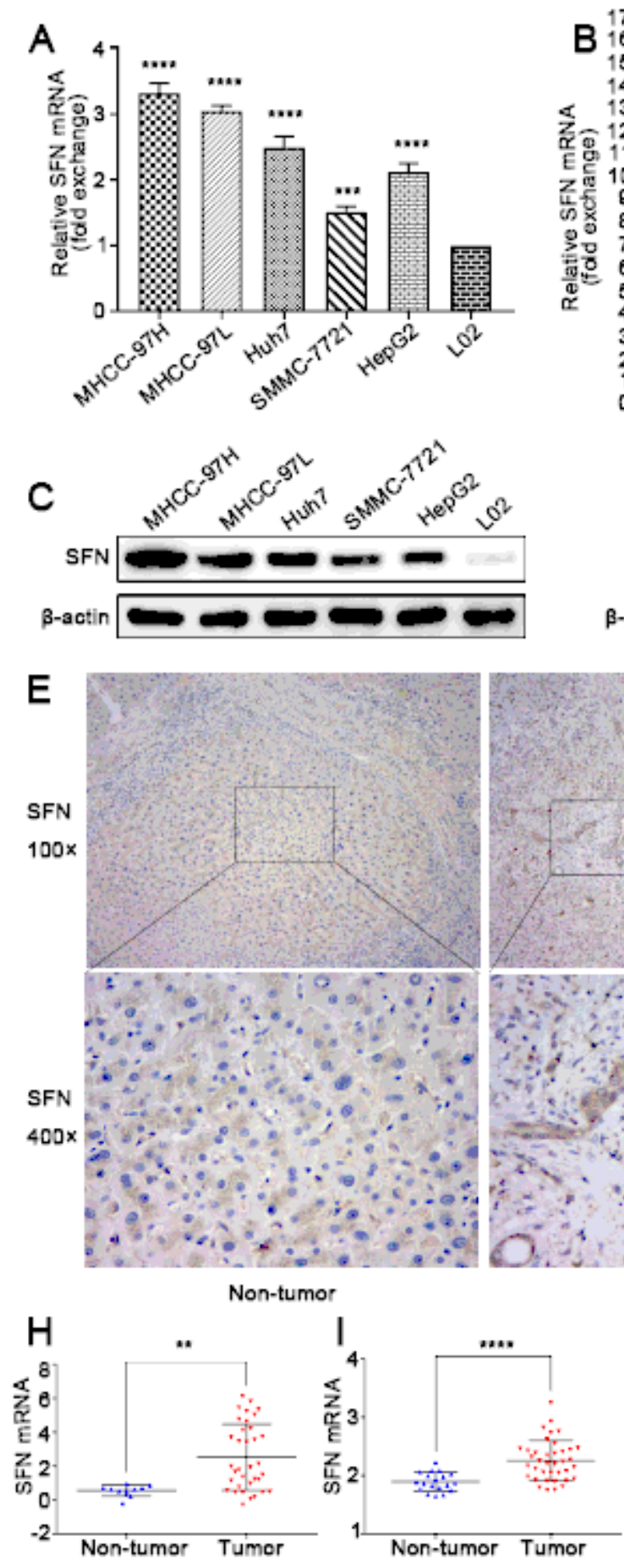
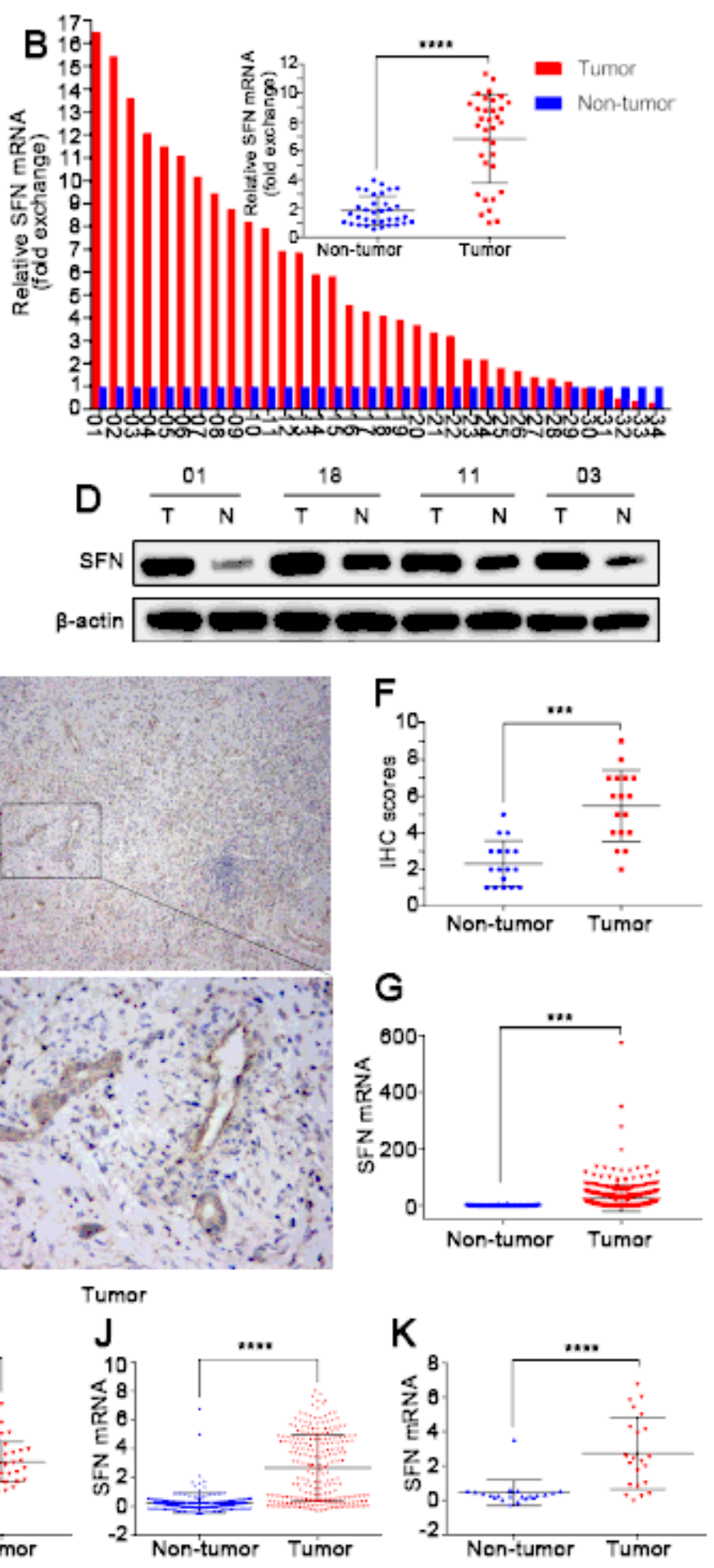

\section{Figure 1}

SFN gene expression is obviously up-regulated in HCC cells and tissues. (A) RT-qPCR identified the mRNA expression levels in six HCC cell lines and L02 cells. (B) SFN mRNA were explored in 34 paired fresh HCC tissues and adjacent non-tumor tissues by RT-qPCR. (C) Western blotting examined the protein expression levels of SFN in six HCC cell lines and L02 cells. (D) SFN protein levels were detected in 34 paired fresh HCC tissues and adjacent non-tumor tissues by RT-qPCR. (E) Part immunohistochemistry (IHC) images of SFN expression in HCC tissues and adjacent non-tumor tissues. (F) The final IHC score between HCC 
tissues and adjacent non-tumor tissues. (G) SFN was up-regulated in 377 HCC cases compared to 50 non-tumor cases by analyzing TCGA dataset. $(\mathrm{H}, \mathrm{I}, \mathrm{J}, \mathrm{K}) \mathrm{SFN}$ was overexpression in HCC tissues in four Oncomine datasets (Roessler liver, Mas liver, Roessler liver 2, Wurmbach liver). ${ }^{\star \star P} \otimes 0.01,{ }^{\star \star \star} \mathrm{P} \otimes 0.001$, $\star * * * P \otimes 0.0001$.
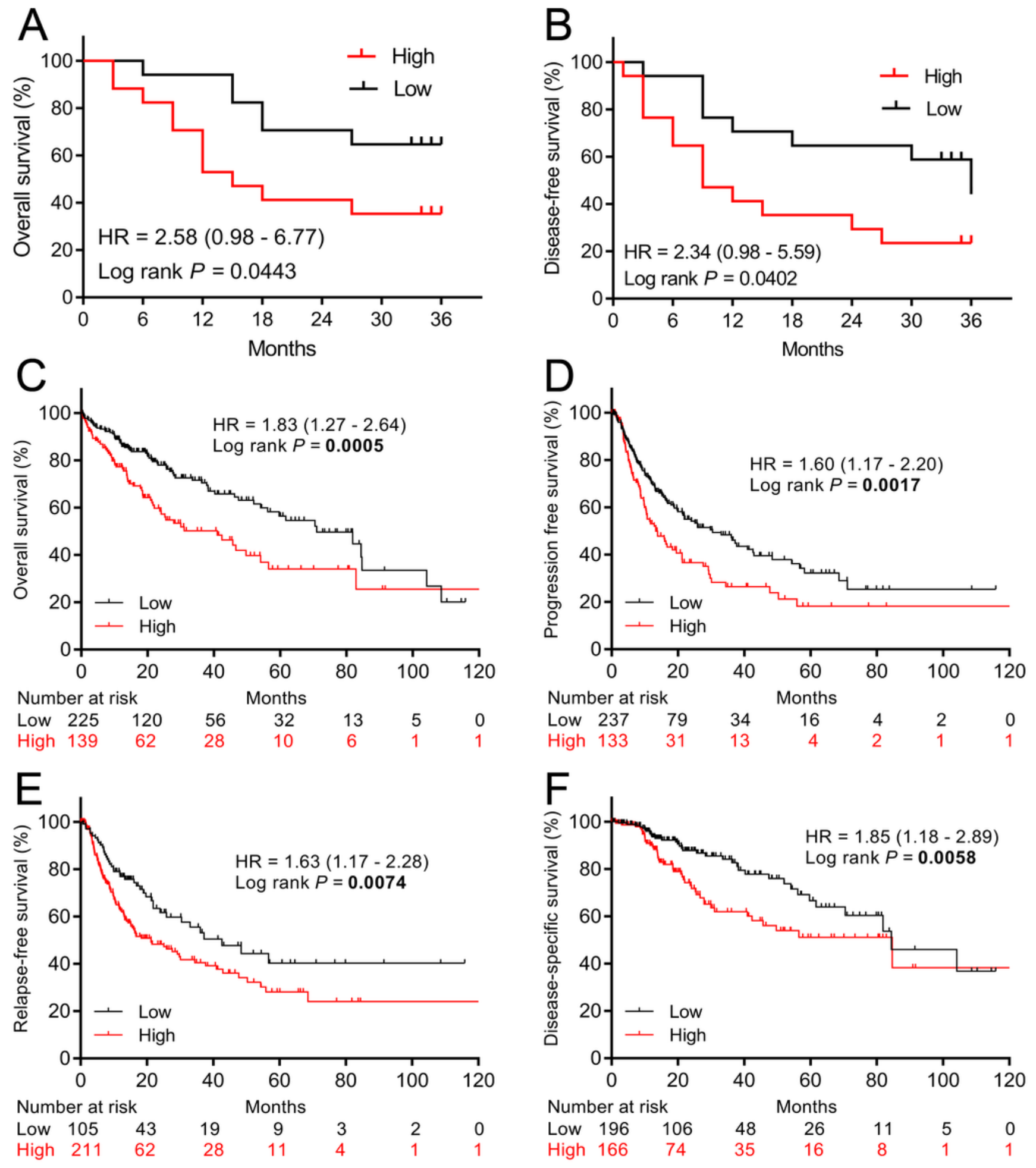

Figure 2 
SFN gene is closely associated with poor prognosis. (A, B) The overall survival (OS) and disease-free survival (DFS) of HCC patients in low or high SFN expression cohort. (C, D, E, F) The OS, progression free survival (PFS), relapse-free survival (RFS), and disease-specific survival (DSS) of HCC cases in the Kaplan-Meier plotter online database.
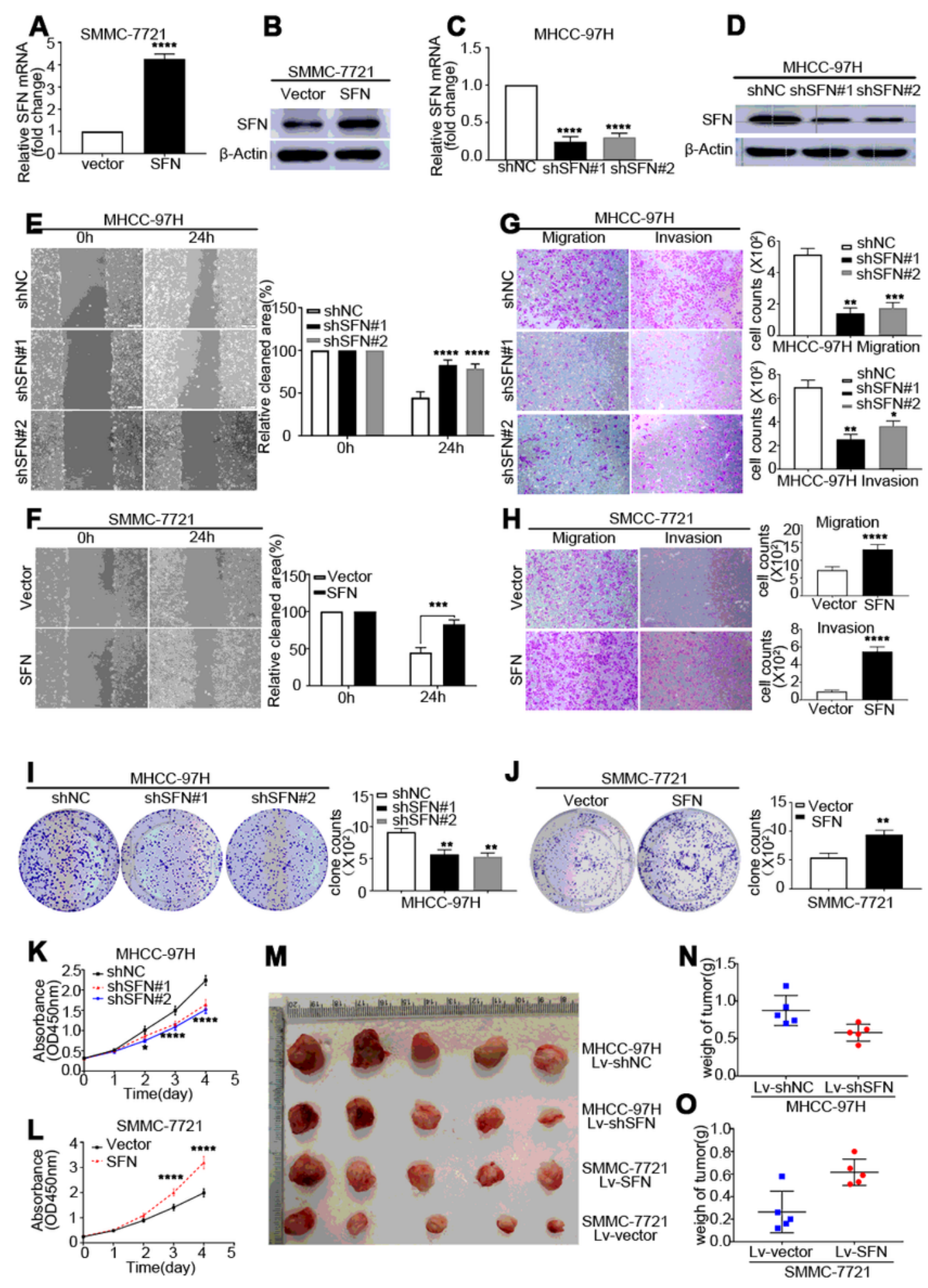

Figure 3 
SFN promotes HCC cells proliferation, migration, invasion, growth in vitro and in vivo. The efficiency of SFN up-regulated or knockdown was validated by RT-qPCR and Western blotting in MHCC-97H (A and B) and SMMC-7721 ( $D$ and $E$ ) cell lines. The CCK-8 assays were used to detect the proliferation capacities of MHCC-97H (C) and SMMC-7721 (F) cells with modified SFN expression. The wound healing assays (G, I) and transwell migration assays $(\mathrm{H})$ were used to explore the migratory abilities of HCC cells with upregulated or down-regulated SFN expression. (J) The invasion capacities of HCC cells with up-regulated or down-regulated SFN expression were examined by transwell invasion assays. (K) The colony formation assays were utilized to investigate the proliferation abilities of HCC cells with changed SFN expression. (L) The xenograft mice models analyzed the role of SFN on tumor growth, images of tumors derived from MHCC-97H with SFN knockdown or SMMC-7721 with SFN overexpression in subcutaneous of nude mice. $(\mathrm{M}, \mathrm{N}, \mathrm{O}, \mathrm{P})$ The weigh and volume of tumor in each xenograft mice models. ${ }^{*} \mathrm{P} \otimes 0.05, * * \mathrm{P} \nabla$ $0.01, * \star * P \otimes 0.001, \star \star \star \star P \otimes 0.0001$. 
A

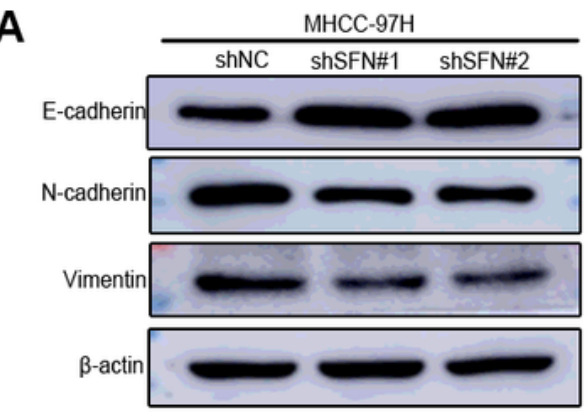

C

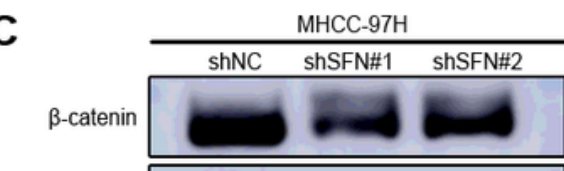

Non-p- $\beta$-catenin (Active)



p-GSK-3
(Ser9)



Axin2

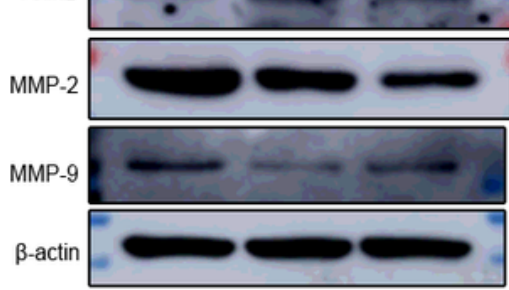

E

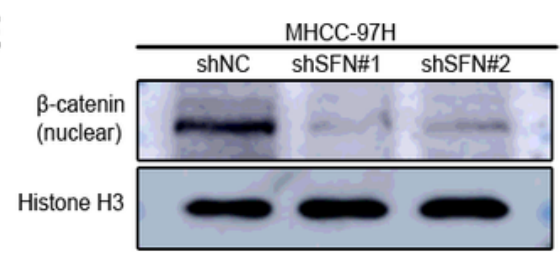

G



B

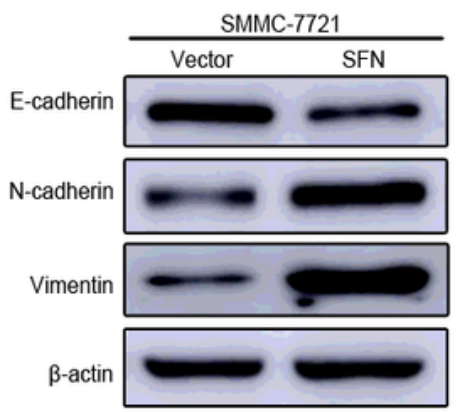

D

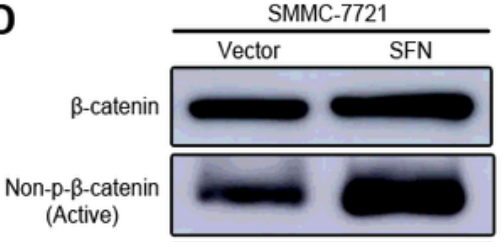
(Active)

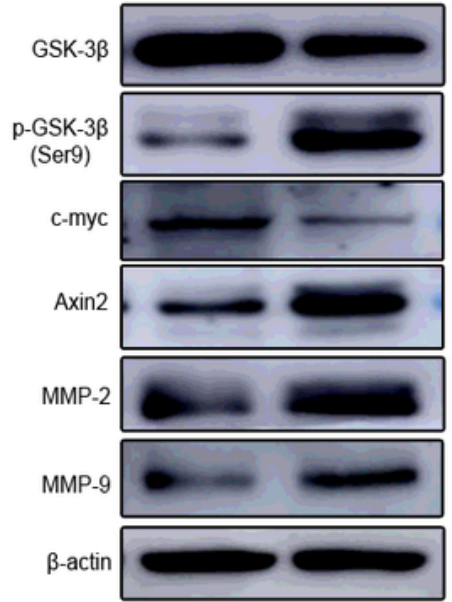

$\mathbf{F}$

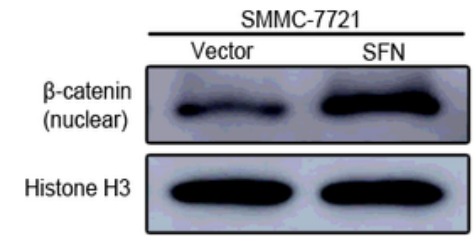

$\mathbf{H}$



\section{Figure 4}

SFN induces EMT and activates Wnt/ $\beta$-catenin signaling in HCC cells. (A) The protein levels of Ecadherin, N-cadherin and vimentin in MHCC-97H cells infected with lentiviruses of shSFN\#1, shSFN\#2 or shNC as detected by western blot. (B)The protein levels of E-cadherin, $\mathrm{N}$-cadherin and vimentin in SMMC7721 cells infected with lentiviruses over-expressing SFN or vector as detected by western blot. (C) The

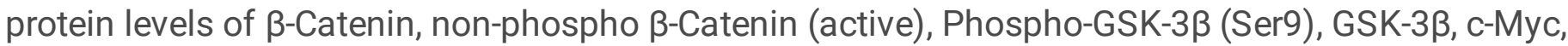


Axin2, MMP-2 and MMP-9 in MHCC-97H cells infected with lentiviruses of shSFN\#1, shSFN\#2 or shNC as detected by western blot. (D) The protein levels of $\beta$-Catenin, non-phospho $\beta$-Catenin (active), PhosphoGSK-3 $\beta$ (Ser9), GSK-3 $\beta$, c-Myc, Axin2, MMP-2 and MMP-9 in SMMC-7721cells infected with lentiviruses over-expressing SFN or vector as detected by western blot. (E)The protein level of $\beta$-Catenin in nuclear of MHCC-97H cells infected with lentiviruses of shSFN\#1, shSFN\#2 or shNC as detected by western blot.

(F)The protein levels of $\beta$-Catenin in nuclear of SMMC-7721 cells infected with lentiviruses overexpressing SFN or vector as detected by western blot.

\section{Supplementary Files}

This is a list of supplementary files associated with this preprint. Click to download.

- SupplementFig1.tif

- Supplementtable1.docx 